\title{
Virtual Human Imaging
}

\author{
Yang Cai ${ }^{1}$, Iryna Pavlyshak ${ }^{1}, \mathrm{Li} \mathrm{Ye}^{1}$, Ryan Magargle ${ }^{2}$, and James Hoburg ${ }^{1}$ \\ ${ }^{1}$ Carnegie Mellon University, Pittsburgh, PA, USA \\ ycai@cmu.edu \\ 2 Ansoft, Inc., Pittsburgh, PA, USA \\ rmagargle@ansoft.com
}

\begin{abstract}
Given 3D scanned anthropological models and the physical parameters of a microwave imaging system, we develop a virtual human surface imagery system with a finite multi-physics surface model. The concealed object detection algorithms are developed based on the wave intensity and surface characteristics. The virtual human image system can be integrated into a systematic design process, enabling multidisciplinary innovations in security, privacy, healthcare, computer vision, and information visualization. This forward-thinking approach intends to transform the development of human imaging technologies from being device-specific and proprietary to being deviceindependent and open source-oriented. It also transforms the research into a systematic design process, enabling multidisciplinary innovations in digital human modeling, computer vision, information visualization, and computational aesthetics. This study can help to design privacy-aware imaging systems in airports and medical systems.
\end{abstract}

Keywords: human body, feature recognition, 3D scan, security, privacy.

\section{Introduction}

The goal of this study is to build a general computational model for designing and evaluating human imaging technologies before a physical system is built. This forward-thinking approach intends to transform the development of human imaging technologies from being device-specific and proprietary to being device-independent and open source. This also transforms imaging research into a systematic design process, which requires multidisciplinary innovations in digital human modeling, computer vision and information visualization.

For example, the growing demand for three-dimensional holographic imaging systems has created significant interests in many disciplines. Current devices operate using a millimeter wave transceiver to reflect the signal off the human body and any objects carried on it. These devices penetrate items that are less dense, such as clothing and hair $[5,6,7,8,9,10,11,12,13,14,15,45]$. Unlike the latest metal detectors, the system can also detect non-metal threats or contraband, including plastics, liquids, drugs and ceramic weapons hidden under clothing. These high-resolution scanned images reveal intimate bodily details and have raised serious privacy concerns.

Most of the research and development of human scanning systems has been done through unpopular projects in a few private companies or in government laboratories. 
As a result, most of the technologies are either device specific or proprietary, which has slowed down the overall advancement of privacy technologies for the $3 \mathrm{D}$ body scanning systems.

The following problems warrant a scientific investigation: 1) Given the available databases of anthropological models and the physical parameters of human imaging systems, we simulate the scanning imagery data to be used as an open source for broader research communities; 2) We develop effective algorithms to find the human surface features from the 3D scanning data; Finally, 3) we develop the algorithms to discern concealed objects from the human body. Fig. 1 shows an illustration of the framework.

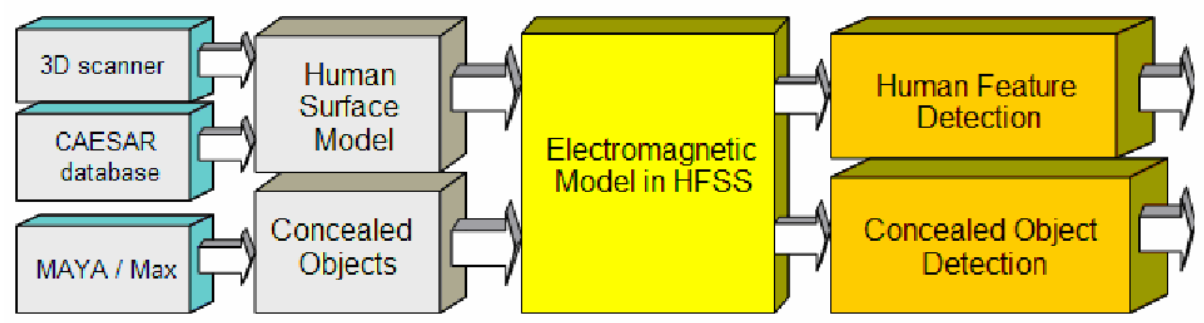

Fig. 1. The framework of the multidisciplinary modeling process that merges at least four domains: computer simulation, computer vision, information visualization and human-centered computing

The physically augmented virtual human model is the central idea in the study. In the world of medical research and development, scientists often use so-called 'phantoms' to calibrate a new medical instrument. Affordable phantom databases and artifacts, such as Mathworks' MRI brain phantom images [33], National Library of Medicine's Visible Humans [34] and DARPA's Digital Soldier [35], significantly reduce development cycles and increase opportunities for interdisciplinary collaboration and education. Currently, there is no shared scientific benchmarking database in the security human scanning area. In this project, we will develop digital human models that not only contain finite surface elements but also physical properties, for example the reflection of microwave beams on the skin and concealed objects beneath clothing. This requires high-fidelity modeling within a high frequency $(900 \mathrm{MHz}$ to $33 \mathrm{GHz})$ electromagnetic field simulation, which reaches the limit of current physical computation technologies. Compared to MRI imaging simulation, this task is more computationally challenging. We envision that our results will inspire a new area of virtual imaging technologies. The difficulties of our proposed project include: mapping the imperfect laser scanning surface data to the finite element material data, formulating the electromagnetic exciting sources, and calibrating the simulated model.

The algorithm for detecting human surface features enables us to segment the human body and reduce the search space for anomalous objects. Many machine learning algorithms are coordinate-dependent and limited by the training data space, for example, artificial neural networks [44]. Some algorithms only work within small bounding boxes that do not deliver an acceptable performance. For example, if a feature detection algorithm takes one hour to process, then it is not useful for a security screening 
system [31,32]. In this project, we want to develop a model that is invariant to poses and coordinates. From a computer vision point of view, detecting features from 3D body scan data is nontrivial because human bodies are diverse. The technical methodology of function fitting has been used for extracting special landmarks, such as ankle joints, from 3D body scan data [31,32], similar to the method for extracting landmarks on terrain [21,22]. Curvature calculation is also introduced from other fields such as the sequence dependent curvature structure of DNA $[19,20]$. These curvature calculations use methods such as chain code [30], circle fit, ratio of end-to-end distance to contour length, ratio of moments of inertia, and cumulative and successive bending angles. Curvature values are calculated from the data by fitting a quadratic surface over a square window and then calculating the directional derivatives of this surface. Sensitivity to data noise is a major problem in both the function fitting and curvature calculation methods because typical 3D scanning data is very noisy. Template matching appears to be a promising method because it is invariant to the coordinate system $[31,32]$. However, defining a template and where to match the template is challenging because it is unique to each particular feature.

How to develop a discriminative algorithm to distinguish anomalous objects from human parts is a challenge. In this study, we focus on surface and density characteristics, where the objects can be clustered and highlighted based on the spatial curvature and spatial density of object data points. Artificial anomalous objects embedded into the realistic $3 \mathrm{D}$ datasets are used to evaluate the performance of the developed algorithms. This task is the most difficult but most important in the project.

\section{Physically Augmented Virtual Human Model}

We have developed a set of full-scale virtual human models based on the digital surface scanning data from CAESAR database (with necessary license agreement), which contains 50 males and 50 females aged 16-65, where 50 of them are North American, 24 are Asian, and 26 are from the European survey of Italy, the Netherlands and other countries. As we know, all models in the database have feature landmarks which are important anthropomorphic measurements. We keep them in our test-bed. However, all models wore tight underwear. Therefore, we have to remove that by applying a low-pass filter.

In addition, we also use the state-of-art high fidelity laser $3 \mathrm{D}$ scanner $^{1}$ to collect our own 20 samples as references. For these in-house models, we will manually annotate the human landmarks. Fig. 2 shows a sample of a 3D human body scanning data and the output of the microwave imaging simulation from $\mathrm{HFSS}^{2}$.

We input the human scan model to High Frequency Simulation System (HFSS) where we assign the microwave reflection properties to the $3 \mathrm{D}$ surface point clouds. This is a non-trivial task because no one has done the full-body microwave imaging simulation with HFSS before. According to state-of-the-art microwave simulation technology, the approach is feasible but very challenging due to the limitations of the

\footnotetext{
${ }^{1}$ www.creaform3d.com

${ }^{2}$ http://www.ansoft.com/products/hf/hfss/
} 
current software and computers. If we succeed, it would bring a brand new direction for virtual prototyping of imagery devices. To accomplish the task within the limited time and budget, we simplify the computing problems. For example, to reduce the mesh resolution to fit the capacity of the HFSS model, we use the snapshots of frequencies instead of frequency sweeping process and model only portions of the body instead of the whole.

We researched the related patents in order to reverse engineer the technical details. We have found at least two kinds of systems, for example, the Ka-band (27 - 33 $\mathrm{GHz})$ device and $\mathrm{Ku}$ band $(12-18 \mathrm{GHz})$ device. Due to the different wavelengths and scanning methods, the returning signals are different. In addition, we add anomalous objects such as concealed weapons like guns and knives. Fig. 2 shows an example of the data with the artificial noises and anomalous artifacts in voxels.

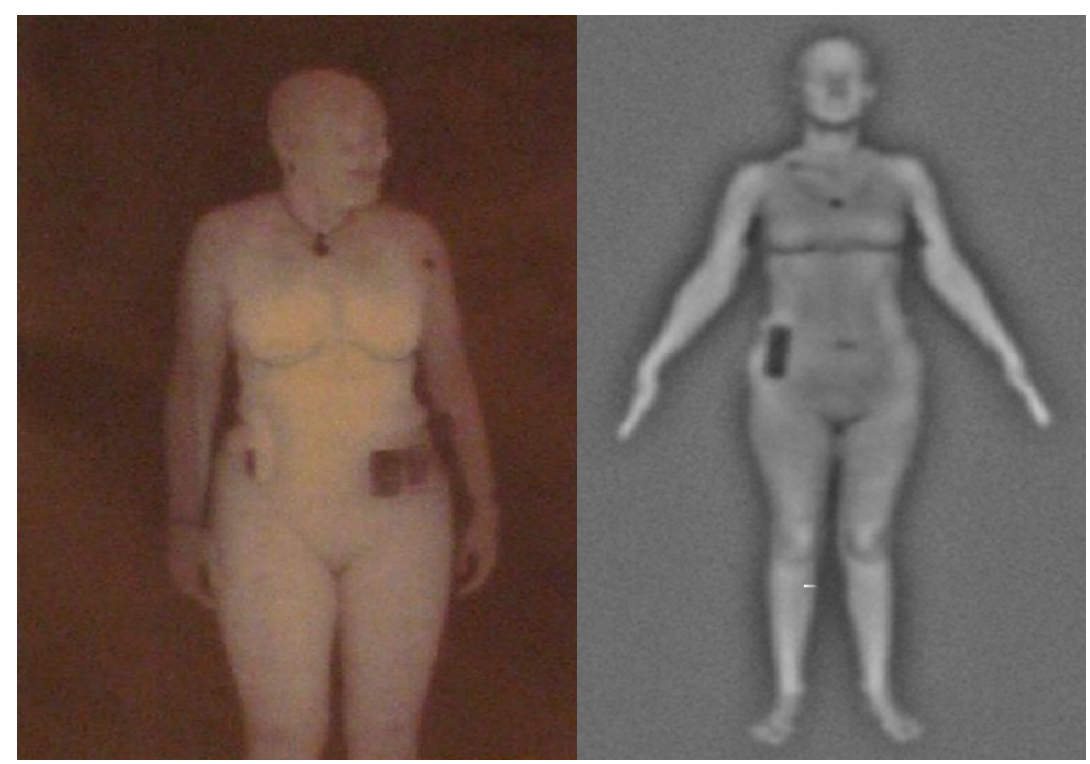

Fig. 2. Real scan image from London Airport (left) and the synthetic image (right)

\section{Algorithm for Detecting Anomalous Objects on Skin}

Effectively detecting anomalous objects and distinguishing them from human body is the ultimate purpose of the system. As a result, it can significantly suppress the human body details as a background. Removing all the human background information may be not desirable because we need the location and size references. There are many anomaly detection methods: bump-hunting, voxel intensity based, curvaturebased and spatial density based clustering, and so on [36-43]. In this project, we develop two spatial analysis models for the anomaly detection: an intensity based detection model and a curvature-based model based detection model. 


\subsection{Intensity-Based Detection}

Metal weapons have their own signatures of intensity properties. However, there are too many kinds of non-metal weapons, such as liquid explosives, which emit different intensity signals. A fixed threshold won't work. In this study, we use HFSS to simulate the scattered radio waves from the objects and human body.

A typical human scanner's wave range is between $500 \mathrm{MHz}$ to $33 \mathrm{GHz}$, which is a great challenge to simulate the whole body imaging at the resolution of $1 \mathrm{~mm}$ with the existing computing resources. To simplify the problem, we crop the 3D human model to a solid $1 \times 1 \times 0.5 \mathrm{ft}^{3}$ slab with a metal gun on the skin. We use the material property for the body with a permittivity and conductivity matching that of sea water (epsilon_r $=81$, and conductivity $=4 \mathrm{~S} / \mathrm{m})$. We have a material for human muscle, but it is only valid up to $6 \mathrm{GHz}$ (epsilon_r $\sim=50$, conductivity $\sim=6 \mathrm{~S} / \mathrm{m}$ ), so we chose to stick with sea water and wouldn't expect a significant difference for the qualitative purposes of this simulation. The gun has the properties of copper. Here is a result for the scattered electric field due to a $1 \mathrm{~V} / \mathrm{m}$ incident plane wave:

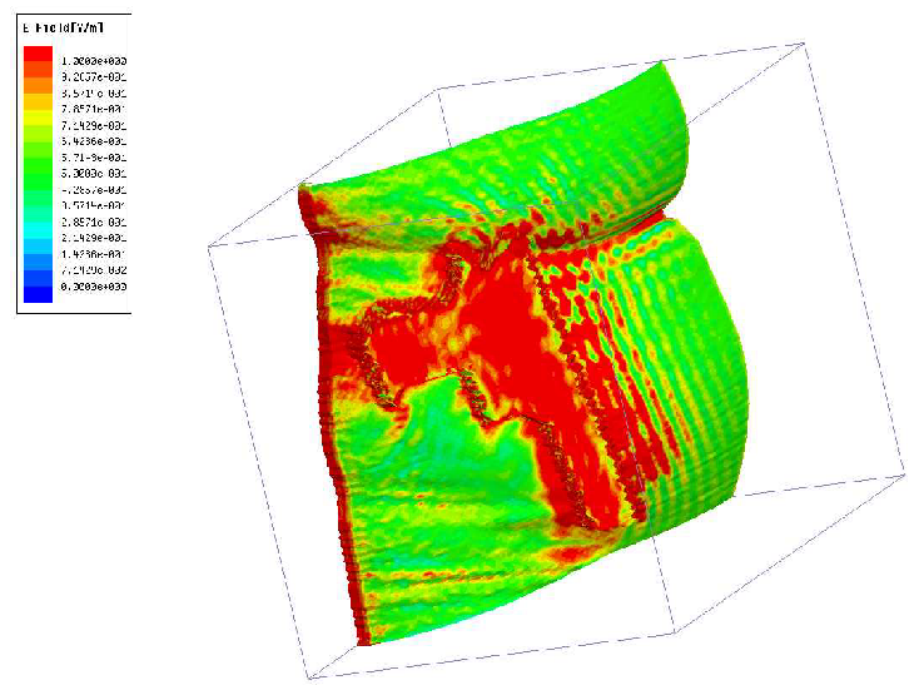

Fig. 3. HFSS simulation of the wave intensive image of human body with a gun at $6 \mathrm{GHz}$

This is the plot of the magnitude of the electric field at a fixed phase. If we plotted for a sequence of phases between 0 and 180, we would see the field magnitude propagate across the body. Note that the area occupied by the gun has magnitude values near the full $1 \mathrm{~V} / \mathrm{m}$, indicating that it reflects most of the signal, whereas the body reflects only around half of that. If we look closely on the left side of the body where it intersects the bounding box, we can see a region of high field value (red). This is due to an artificial resonance with the gun and the boundary condition on the box. For qualitative purposes, the effect on the rest of the simulation should localized and negligible. 
Based on the simulated image samples of known materials, we can train an artificial neural network (e.g. Radial Basis Function) to recognize the signature intensity [30]. To adapt to a broader range of data and a greater noise level, we will preprocess the data with normalization algorithms and filters.

\subsection{Surface Based Detection}

In the continuous case, curvature is defined as the rate of change of slope. In our case, the discrete space, the curvature description must be slightly modified to overcome difficulties resulting from violation of curve smoothness.

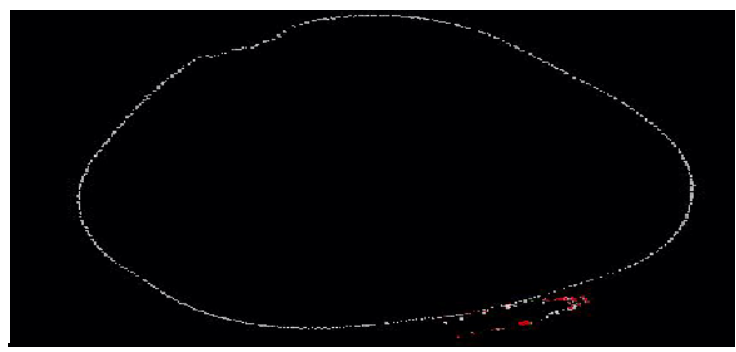

Fig. 4. Slice based feature detection (the object is colored in red)

We start by slicing the digital model horizontally. We average the points between the slices. The curvature scaler descriptor here finds the ratio between the total number of boundary pixels (length) and the number of boundary pixels where the boundary direction changes significantly. The smaller the number of direction changes, the straighter the boundary. In this case, we map the points on the slice to a polar coordinate system because the shape of a body cross-section is in an oval shape.

With $n$ points, the coordination transforms are defined by equations (1)-(2):

$$
\begin{gathered}
r=\sqrt{\left(x-\frac{1}{n} \sum_{i=1}^{n} x_{i}\right)^{2}+\left(y-\frac{1}{n} \sum_{i=1}^{n} y_{i}\right)^{2}} \\
\theta=\arccos \left(\frac{x-\frac{1}{n} \sum_{i=1}^{n} x_{i}}{r}\right) \quad \text { when } \quad y>\frac{1}{n} \sum_{i=1}^{n} y_{i} \\
\theta=\pi+\arccos \left(-\frac{x-\frac{1}{n} \sum_{i=1}^{n} x_{i}}{r}\right) \quad \text { when } \quad y<\frac{1}{n} \sum_{i=1}^{n} y_{i}
\end{gathered}
$$

Then we use a function of the radius and angles of the points to calculate the anomalous features. From Figure 4, we can see that the anomalous increased the point intensity of surface contour. So the intensity-based method calculates the average point distance of specified number of neighboring points. If the neighborhood average 
distance exceeds the product of overall average distance and specified ratio, then the neighboring local area was marked as the anomalous. The algorithm is presented below:

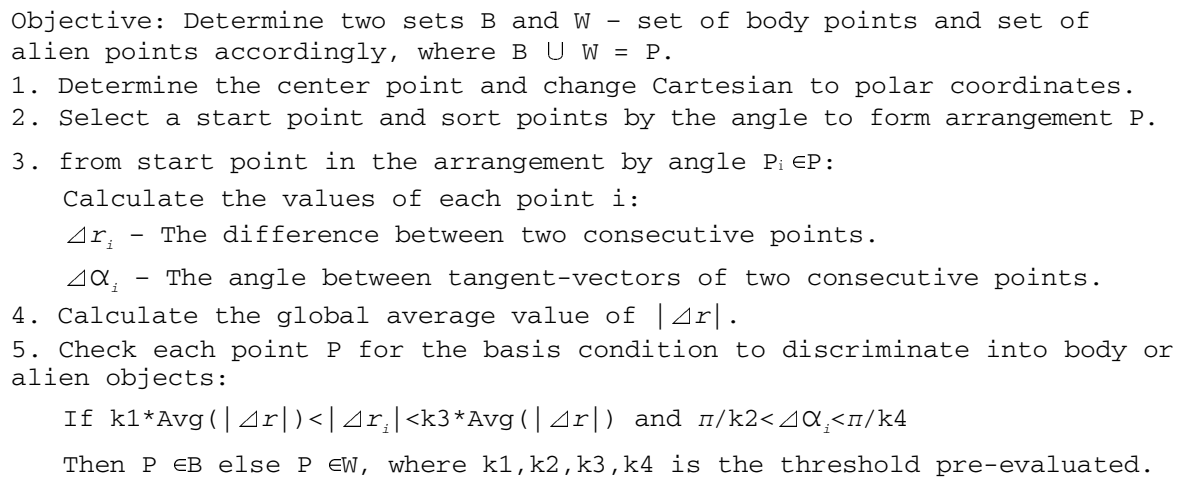

The surface based detection was base on the surface curvature calculation. It is well known how the notion of Gaussian curvature extends to such discrete surfaces $S$ which formed by triangle facets. Thus the Gaussian curvature is supported on the vertices $p \in S$. Its numerical value is the product of the principal curvatures, $\kappa_{1}$ and $\kappa_{2}$, of the given point. From the concept of Discrete Differential Geometry, the curvature is calculated by the following equation:

$$
K(p)=\frac{1}{A(p)}\left(2 \pi-\sum_{p_{i} \in N(p)} \theta_{i}\right)
$$

where $A(p)$ is the sum of surface area of triangle facet of the vertex point $p$, and $\theta$ is the angle of the corresponding facet.

The curvature of each point is calculated by the following equation [17]:

$$
K(p)=\frac{\dot{x} \cdot \ddot{y}-\ddot{x} \cdot \dot{y}}{\left(\dot{x}^{2}+\dot{y}^{2}\right)^{3 / 2}}
$$

where $\dot{x} \cdot \dot{y}, \ddot{x} \cdot \ddot{y}$ represented the first-order and second-order differential. For the sequenced discrete points, we use the difference to replace the differential. The interval expressed by curvatures which exceeds the specified threshold illuminates the anomalous. Fig. 5 shows the final detection results.

We believe that fusion of the intensity-based detection and surface based detection will improve the feature detection accuracy and reduce the noise. Because we have the heterogeneous human models with different resolution and different orientations and sizes, model registration appears to be a challenge. However, for the actual human scanning systems, this is not a problem because the coordinates of the point clouds are known to the designers. 

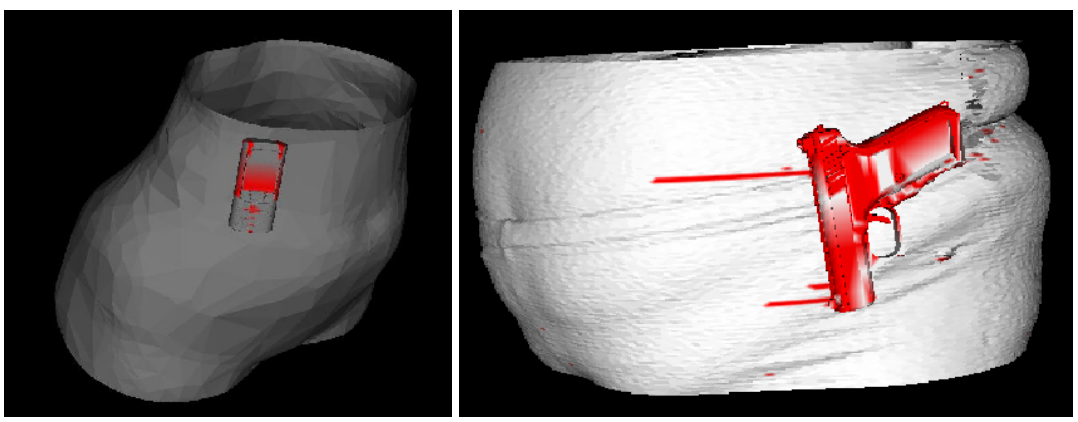

Fig. 5. Detected mobile phone object (left) and highlighted gun with the surface feature (right)

\section{Conclusions}

The goal of the proposed research is to build a virtual human imaging system for designing and evaluating the related technologies before a physical system is built. Given the available databases of anthropological models from CAESAR, 3D scanners and the physical parameters of human imaging systems, we simulate the scanning imagery data with High Frequency Simulation System (HFSS).

The concealed object detection algorithms are developed based on the wave intensive and surface characteristics. This forward-thinking approach intends to transform the development of human imaging technologies from being device-specific and proprietary to being device-independent and open source-oriented. It will also transform the research into a systematic design process, enabling multi-disciplinary innovations in digital human modeling, computer vision, information visualization and computational aesthetics.

The result of this project would have impacts on privacy-aware imaging systems in airports and medical systems. They can also benefit custom-fit products that are designed from personal 3D scanning data. Our results can be used in the reconstruction of ancient artifacts in digital archeology. In addition, they can be applied to medical diagnoses and procedures, such as virtual colonoscopy.

\section{Acknowledgement}

We would like to thank for National Science Foundation for the Cyber Trust grant CT-ER 0716657 and ARO for the research grant for security technologies. Many thanks to Joseph Laws, Emily Hart, Nathaniel Bauernfeind, and Brian Zeleznik for their participation of the project. We would also appreciate the help from Alva Karl of Air Force for the CAESER database.

\section{References}

1. Law, J., Cai, Y.: Feature Hiding in 3D Human Body Scans. Journal of Information Visualization 5(4) (2006)

2. Laws, J., Cai, Y.: A Privacy Algorithm for 3D Human Body Scans. In: Alexandrov, V.N., van Albada, G.D., Sloot, P.M.A., Dongarra, J. (eds.) ICCS 2006. LNCS, vol. 3994, pp. 870-877. Springer, Heidelberg (2006) 
3. Cai, Y., et al.: Spatiotemporal data mining for tracking ocean objects. In: Proceedings of IEEE Space Mission Challenges to IT, Pasadena, CA (2006)

4. Cai, Y., et al.: Visual Transform for spatiotemporal data mining. Journal of Knowledge and Information Systems (to appear) (2007)

5. BodySearch imaging system, American Science and Engineering, Inc., 829 Middlesex Turnpike, Billerica, MA 01821 (2007)

6. Secure 1000 imaging system, IRT Corporation, 6020 Cornerstone Court West, San Diego, CA 92121 (2007)

7. McMakin, D.L., Sheen, D.M., Collins, H.D., Hall, T.E., Severtsen, R.H.: Wideband, millimeter-wave, holographic surveillance systems. In: EUROPTO International Symposium on Law Enforcement Technologies: Identification Technologies and Traffic Safety, Munich, FRG, SPIE, vol. 2092, pp. 131-141 (1995)

8. Sheen, D.M., McMakin, D.L., Collins, H.D.: Circular scanned millimeter-wave imaging system for weapon detection. In: EUROPTO International Symposium on Law Enforcement Technologies: Identification Technologies and Traffic Safety, Munich, FRG, SPIE, vol. 2092, pp. 122-130 (1995)

9. McMakin, D.L., Sheen, D.M., Collins, H.D., Hall, T.E., Smith, R.R.: Millimeter-wave, high-resolution, holographic surveillance system. In: EUROPTO International Symposium on Substance Identification Technologies, Innsbruck, Austria, SPIE, vol. 2092, pp. 525535 (1993)

10. Sheen, D.M., McMakin, D.L., Collins, H.D., Hall, T.E.: Weapon detection using a wideband millimeter-wave linear array imaging technique. In: EUROPTO International Symposium on Substance Identification Technologies, Innsbruck, Austria, SPIE, vol. 2092, pp. 536-547 (1993)

11. Huguenin, G.R., Goldsmith, P.F., Deo, N.C., Walker, D.K.: Contraband Detection System, U. S. Patent 5,073,782 (1991)

12. Browne, J.: MM waves aid commercial applications, Microwaves and RF, pp. 113-116 (July 1992)

13. Goodman, J.W.: Introduction to Fourier Optics. McGraw-Hill, New York (2005)

14. Soumekh, M.: Bistatic synthetic aperture radar inversion with application in dynamic object imaging. IEEE Transactions on Signal Processing 39(9), 2044-2055 (1991)

15. Soumekh, M.: Fourier Array Imaging. Prentice Hall, Englewood Cliffs (1994)

16. Anthropometry Resource (CAESAR), Final Report, Volume I: Summary, AFRL-HE-WPTR-2002-0169, United States Air Force Research Laboratory, Human Effectiveness Directorate, Crew System Interface Division, 2255 H Street, Wright-Patterson AFB OH 45433 7022 and SAE International, 400 Commonwealth Dr., Warrendale, PA 15096 (2007)

17. Jalba, A.C., Wilkinson, M.H.F., Roerdink, J.: Shape Representation and Recognition Through Morphological Curvature Scale Spaces. IEEE Trans. Image Processing 15(2), 331-341 (2006)

18. Forsyth, D.A., Fleck, M.M.: Automatic detection of human nudes. International Journal of Computer Vision 32(1), 63-77 (1999)

19. Forsyth, D.A., Fleck, M.M.: Body Plans. In: Proc. CVPR 1997, pp. 678-683 (1997)

20. Forsyth, D.A., Fleck, M.M.: Identifying nude pictures. In: Proceeding Third IEEE Workshop on Applications of Computer Vision, pp. 103-108 (1996)

21. Goldgof, D.B., Huang, T.S., Lee, H.: Feature extraction and terrain matching. In: Proc. IEEE Comput. Soc. Conf. Comput. Vision Pattern Recognition, Ann Arbor, MI (May 1988)

22. Goldgof, D.B., Huang, T.S., Lee, H.: A Curvature-Based Approach to Terrain Recognition, vol. 11(11), pp. 1213-1217 (November 1989) 
23. Gordon, G.: Face recognition based on depth and curvature features. In: Proceedings of the IEEE Computer Society Conference on Computer Vision and Pattern Recognition (Champaign Illinois), pp. 108-110 (1992)

24. Li, P., Corner, B.D., Paquette, S.: Evaluation of a surface curvature based landmark extraction method for three dimensional head scans. In: International Ergonomics Conference, Seoul (2003)

25. Liu, X., Kim, W., Drerup, B.: 3D Characterization and Localization of Anatomical Landmarks of the Foot. In: Proceeding (417), Biomedical Engineering. Acta Press (2004)

26. Fleck, M.M., Forsyth, D.A., Bregler, C.: Finding naked people. In: Buxton, B., Cipolla, R. (eds.) ECCV 1996. LNCS, vol. 1065, pp. 593-602. Springer, Heidelberg (1996)

27. Ratner, P.: 3-D human modeling and animation. John Wiley \& Sons, Inc., Chichester (2003)

28. Robinette, K.M., Blackwell, S., Daanen, H.A.M., Fleming, S., Boehmer, M., Brill, T., Hoeferlin, D., Burnsides, D.: Civilian American and European Surface Anthropometry Resource (2002)

29. Ioffe, S., Forsyth, D.A.: Probabilistic methods for finding people. International Journal of Computer Vision 43(1), 45-68 (2001)

30. Sonka, M., et al.: Image processing, analysis and machine vision, PWS Publishing (1999)

31. Suikerbuik, C.A.M.: Automatic Feature Detection in 3D Human Body Scans. Master thesis INF/SCR-02-23, Institute of Information and Computer Sciences. Utrecht University (2002)

32. Suikerbuik, R., Tangelder, H., Daanen, H., Oudenhuijzen, A.: Automatic feature detection in 3D human body scans. In: Proceedings of SAE Digital Human Modeling Conference, 04-DHM-52 (2004)

33. Mathworks MRI Phantom (2008), http: / / www. mathworks.com/matlabcentral/fileexchange/ loadFile.do?objectId=1759\&objectType=file

34. NLM, Visible Human Project (2008), http://www.nlm.nih.gov/research/visible/visible_human.html

35. DARPA Virtual Soldier (2008), http://www.wired.com/news/medtech/0,1286,60016,00.html

36. Neill, D.B., Moore, A.W.: Anomalous spatial cluster detection. In: Proc. KDD 2005 Workshop on Data Mining Methods for Anomaly Detection, pp. 41-44 (2005)

37. Neill, D.B., Moore, A.W.: Rapid detection of significant spatial clusters. In: Proc. 10th ACM SIGKDD Conf. on Knowledge Discovery and Data Mining, pp. 256-265 (2004)

38. Salvador, S., Chan, P.: Fastdtw: Toward accurate dynamic time warping in linear time and space. In: KDD Workshop on Mining Temporal and Sequential Data (2004)

39. Shyu, M.L., Chen, S.C., Sarinnapakorn, K., Chang, L.W.: A novel anomaly detection scheme based on principal component classifier. In: Proceedings of the IEEE Foundations and New Directions of Data Mining Workshop (2003)

40. Zhang, J., Zulkernine, M.: Anomaly Based Network Intrusion Detection with Unsupervised Outlier Detection. In: Symposium on Network Security and Information Assurance Proc. of the IEEE International Conference on Communications (ICC), Istanbul, Turkey (June 2006)

41. Burbeck, K., Tehrani, S.N.: ADWICE: Anomaly Detection with Real-time Incremental Clustering. In: Park, C.-S., Chee, S. (eds.) ICISC 2004. LNCS, vol. 3506, pp. 407-424. Springer, Heidelberg (2005) 
42. Gomez, J., Gonzalez, F., Dasgupta, D.: An Immuno-Fuzzy Approach to Anomaly Detection. In: the proceedings of the 12th IEEE International Conference on Fuzzy Systems (FUZZIEEE), May 25-28, 2003, vol. 2, pp. 1219-1224 (2003)

43. Wise, J.A., Thomas, J.J., Pennock, K., Lantrip, D., Pottier, M., Schur, A., Crow, V.: Visualizing the non-visual: spatial analysis and interaction with information from text documents. In: Proceedings of the 1995 IEEE Symposium on Information Visualization, Atlanta, Georgia, October 30-31, 1995, p. 51 (1995)

44. Keller, P., McMkin, L., Sheen, D., McKinnon, A., Summet, A.J.: Privacy Algorithm for Cylindrical Holographic Weapons Surveillance Systems. In: Proc. SPIE, Applications and Science of Computational Intelligence III, vol. 4055, pp. 476-483 (2000)

45. Sheen, D.M., et al.: Concealed explosive detection on personnel using a wideband holographic millimeter-wave imaging system. In: Proceedings of the SPIE, AEROSENSE Conference, Orlando, FL, vol. 2755 (1996) 